\title{
Cytoskeletal heart-enriched actin-associated protein (CHAP) is expressed in striated and smooth muscle cells in chick and mouse during embryonic and adult stages
}

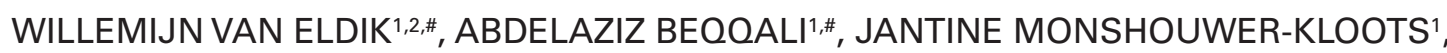 \\ CHRISTINE MUMMERY', ROBERT PASSIER*,1 \\ ${ }^{1}$ Leiden University Medical Center, Department of Anatomy and Embryology, Leiden and
${ }^{2}$ Interuniversity Cardiology Institute of the Netherlands (ICIN), Utrecht, The Netherlands
}

\begin{abstract}
We recently identified a new Z-disc protein, CHAP (Cytoskeletal Heart-enriched Actinassociated Protein), which is expressed in striated muscle and plays an important role during embryonic muscle development in mouse and zebrafish. Here, we confirm and further extend these findings by (i) the identification and characterization of the CHAP orthologue in chick and (ii) providing a detailed analysis of CHAP expression in mouse during embryonic and adult stages. Chick CHAP contains a PDZ domain and a nuclear localization signal, resembling the human and mouse CHAPa. CHAP is expressed in the developing heart and somites, as well as muscle precursors of the limb buds in mouse and chick embryos. CHAP expression in heart and skeletal muscle is maintained in adult mice, both in slow and fast muscle fibers. Moreover, besides expression in striated muscle, we demonstrate that CHAP is expressed in smooth muscle cells of aorta, carotid and coronary arteries in adult mice, but not during embryonic development.
\end{abstract}

KEY WORDS: smooth muscle, skeletal muscle, heart, z-disc, adult, vascular

The Z-disc delineates the borders of the sarcomere, the contractile unit of striated muscle and represents an anchoring plane for various proteins. In addition to their role in force transmission, Zdisc proteins may also be involved in signal transduction (Lange et al., 2006). Recently, we indentified a novel Z-disc protein, which we named CHAP (Cytoskeletal Heart-enriched Actin-associated Protein) (Beqqali et al., 2006, 2010). We have shown that CHAP has two isoforms, $\mathrm{CHAPa}$ and $\mathrm{CHAPb}$, in both humans and mice. CHAPa, the longest isoform (978 amino acids (aa)) contains an $\mathrm{N}$-terminal PDZ domain, as well as a nuclear localization signal (NLS). The shorter CHAPb isoform (749 aa) lacks the PDZ domain, but still contains the NLS. CHAP interacts with $\alpha$-actinin-2, another Z-disc protein, and is able to translocate to the nucleus (Beqqali et al., 2010). Previously, we have shown that ChapA is predominantly expressed in adult heart and muscle tissues, whereas $C h a p B$ is expressed at higher levels in striated muscles during embryonic development. ChapB expression in mouse is evident from the cardiac crescent stage (E7.5) onwards. During later embryonic stages $C h a p B$ expression is maintained in the developing heart, but is also expressed in the somites (giving rise to skeletal muscle). In addition, we have identified the zebrafish Chap orthologue and demonstrated by morpholino antisense oligonucleotide-mediated knockdown that Chap is essential for zebrafish heart and muscle development. Knockdown of Chap in the zebrafish resulted in aberrant muscle development, indicated by defects in cardiac looping, formation of pericardial oedema and disorganized sarcomeres (Beqqali et al., 2010).

Here, we identified the chick (Gallus gallus) orthologue of CHAP. We show that CHAP gene and protein resembles the human and mouse CHAPa isoform, with the predicted PDZ and NLS domains. A detailed analysis of CHAP mRNA and protein expression during development in chick and mouse embryos and in adult tissues is shown. Furthermore, we demonstrate that CHAP is not only expressed in cardiomyocytes, and slow and fast skeletal muscle, but interestingly also in smooth muscle cells of the cardiovascular lineage.

Abbreviations used in this paper: ASMA, $\alpha$-smooth muscle actin; CHAP, Cytoskeletal Heart-enriched Actin-associated Protein; HH, Hamburger and Hamilton; NLS, nuclear localization signal; OFT, outflow tract.

*Address correspondence to: Robert Passier. Einthovenweg 20, 2333 ZC Leiden,The Netherlands. Fax: +31-71-526-8289. E-mail: r.passier@lumc.nl

\# Note: Both authors contributed equally to this work.

Accepted: 23 February 2011. Final, author-corrected PDF published online: 2 September 2011.

ISSN: Online 1696-3547, Print 0214-6282 


\section{Results}

After BLAST search using full-length sequences of the previously identified CHAP orthologues (zebrafish, mouse, and human) (Beqqali et al., 2010), we identified the chick (Gallus gallus, Gg) orthologue of CHAP (GenBank accession no. XM_421618). For amplification, cloning and sequencing of chick $C H A P$ we amplified the predicted full-length open reading frame (ORF) from hearts of chick embryos (HH19, 23 and 27), which resulted in a 3243 bp PCR product. After sequencing we found that the 1081 aa predicted chick CHAP protein (Fig. 1C) had a $48 \%$ and $47 \%$ homology with human (Hs) and mouse (Mm) CHAPa, respectively (Fig. 1A). Furthermore, there was a $43 \%$ and $40 \%$ homology with zebrafish Chap-1 (DrChap-1) and Chap-2 (DrChap-2), respectively (Fig. 1A). The NLS and PDZ domain are conserved between the different species. The chick PDZ domain of CHAP showed $63 \%$ and $61 \%$ homology with the PDZ domains of human and mouse, respectively. The CHAP gene is mapped on chromosome 6 and consists of four exons (Fig. 1B). Similar to zebrafish chap, no chick CHAPb (lacking the PDZ-domain) was identified. In addition to homology of sequence between different species, we also observed conserved

\section{A}

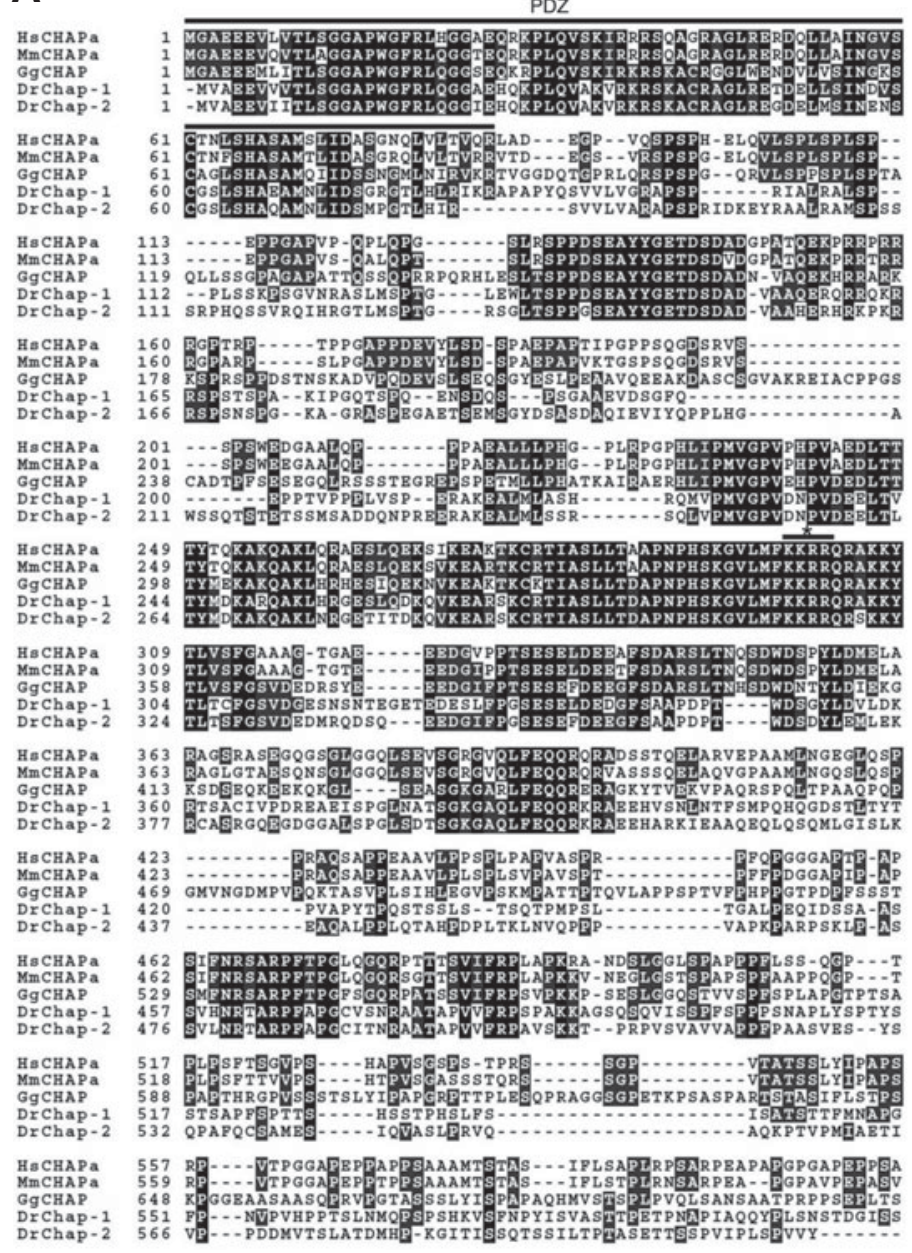

syntenic organization. For instance, myozenin-1, another z-disc protein, is located immediately downstream of the mouse Chap gene (Beqqali et al., 2010). A similar genomic organization was found in the chick genome.

\section{Expression of CHAP during development in chick and mouse embryos}

To investigate the expression pattern of chick CHAP during development we performed whole-mount in situ hybridization. Expression of $\mathrm{CHAP}$ could first be detected at stage $\mathrm{HH} 8$ in the cardiac crescent (Fig. 2A). At $\mathrm{HH} 10$ and $\mathrm{HH} 11$ CHAP was expressed in the linear and looping heart tube (Fig. 2 B,C). CHAP could be detected in the ventricle and outflow tract (OFT), but not in the sinus venosus. At stage $\mathrm{HH} 13, \mathrm{CHAP}$ was strongly expressed in the looping heart (Fig. 2D). From HH15 onward CHAP was expressed in the heart and somites (Fig. 2E), with higher expression in the anterior somites, which are more mature than the posterior somites. At HH17 (Fig. 2F) CHAP was also expressed in the more posterior somites, suggesting that the expression increases as the somites mature. At stage HH17 (Fig. 2F) and stage HH21 (Fig. 2 $\mathrm{G}, \mathrm{H}) \mathrm{CHAP}$ expression was maintained in the heart and somites.

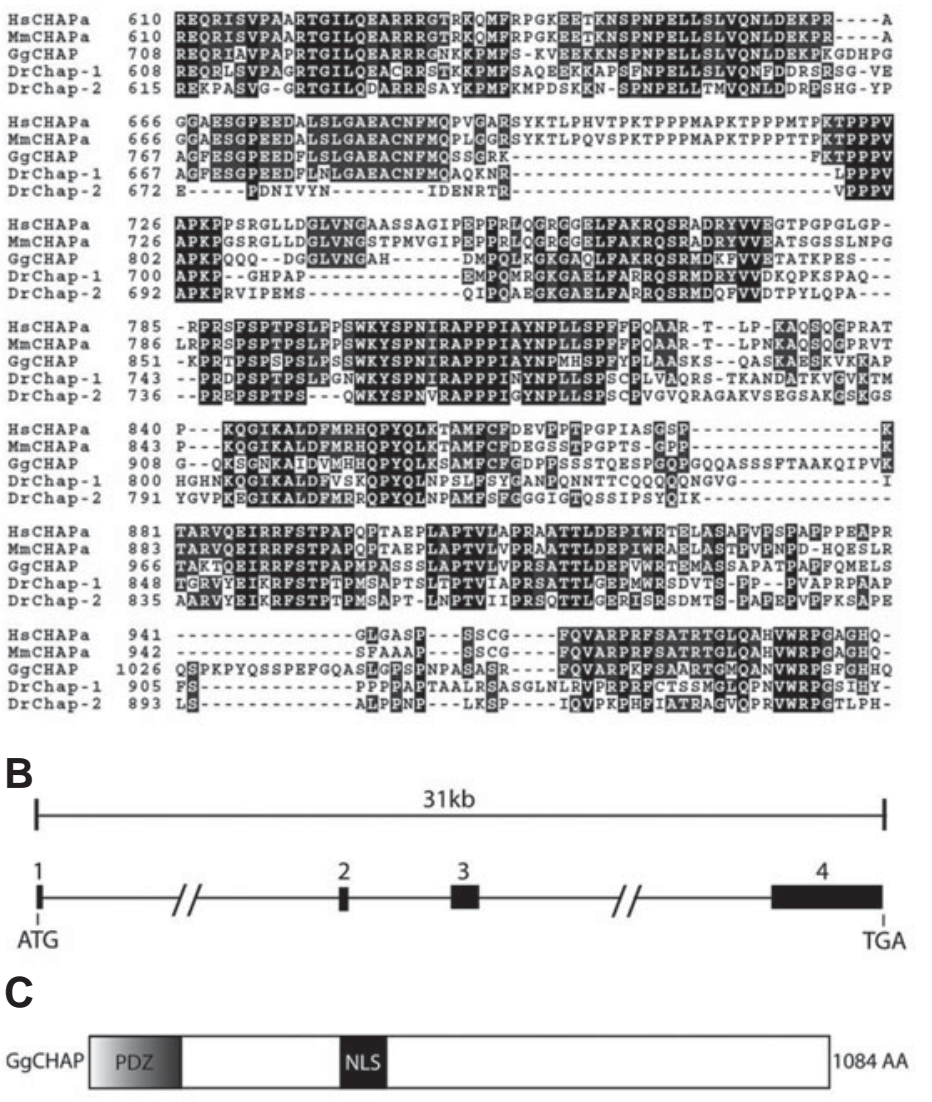

Fig. 1. Sequence and genomic organization of chick CHAP. (A) Alignment and amino acid comparison of Homo sapiens (Hs), Mus musculus (Mm) CHAPa, Gallus gallus (Gg) and Danio rerio (Dr) CHAP. Identical amino acids are shown in black, similar amino acids are gray and gaps are represented by a dash. PDZ and NLS $\left(^{*}\right)$ domains are indicated. (B) Genomic organization of chick CHAP gene. ATG represents start codon, TGA represents stop codon and exons (black boxes) are numbered. (C) Schematic representation of chick (Gg) CHAP protein showing the location of the PDZ and NLS domains. 
At stage $\mathrm{HH} 25$ CHAP was expressed in the muscle precursors of the limb buds, heart and somites (Fig. 2 I,J). The expression of CHAP in muscle precursors of the limb buds of the developing legs and wings was more pronounced in embryos in stages $\mathrm{HH} 28$ 30 (Fig. $2 \mathrm{~K}, \mathrm{~N}, \mathrm{O}$ ). Hearts of $\mathrm{HH} 30$ showed a high expression of $C H A P$ in both atria and ventricles, but not in the OFT (Fig. 2 L,M). Comparison of expression pattern of $C H A P$ during early embryonic development with that of mouse and zebrafish, demonstrate that the CHAP expression pattern is conserved between these species (Beqqali et al., 2010).

To study CHAP expression during chick development in more detail, we performed section in situ hybridization at different stages. At stage $\mathrm{HH} 18$ and $\mathrm{HH} 26$ CHAP was expressed in heart muscle cells and the myotome part of the somites (Fig. $3 \mathrm{~A}-\mathrm{C}$ ), confirming the results of whole-mount in situ hybridization. Occasionally, higher levels of $C H A P$ could be identified at the border with the dermatome, suggesting that CHAP is expressed in more mature cells of the somites, which is also in agreement with the higher expression of $C H A P$ in more anterior somites. At stage $\mathrm{HH} 30$ expression of $C H A P$ was localized in ventricles and atria, whereas endocardial cushions are negative for $C H A P(F i g .3 D, G)$. Furthermore, trunk muscle masses (Fig. $3 \mathrm{E}, \mathrm{H})$, muscle groups from the limb buds of the developing wings (Fig. 3F), tongue, eyes and jaws show CHAP expression (Fig. 3I).

To compare CHAP expression in chick and mouse embryos, we performed in situ hybridization on sagittal sections of E13.5 mouse embryos, using a probe that recognizes both ChapA and ChapB isoforms. As expected, expression of Chap was pronounced in the ventricles and atria of the heart (Fig. 4 A,B). Furthermore Chap was also expressed in the developing muscles of the tongue, jaw, limb buds and tail (Fig. 4A). Detection of Chap was considered to be expression of $C h a p B$, since we have previously demonstrated

Fig. 2. Whole mount in situ hybridization of chick CHAP during development. (A-O) Whole mount in situ hybridization with digoxigeninUTP labeled chick CHAP riboprobe on different developmental stages of chick embryos: HH8 (A), HH1O (B), HH11 (C), HH13 (D), HH15 (E), HH17 (F), HH21 (G-H), HH25(I-J), HH28(K), HH30 heart (L-M), HH3O leg (N) and HH3O wing (O). Cardiac crescent (cc), ventricle (v), atrium (a) and outflow tract (oft), sinus venosus (sv), heart (h), somites (s), muscle precursors (arrow head) of the limb buds (Ib) left ventricle (Iv), right ventricle ( $r v)$, left atrium (la), right atrium (ra).
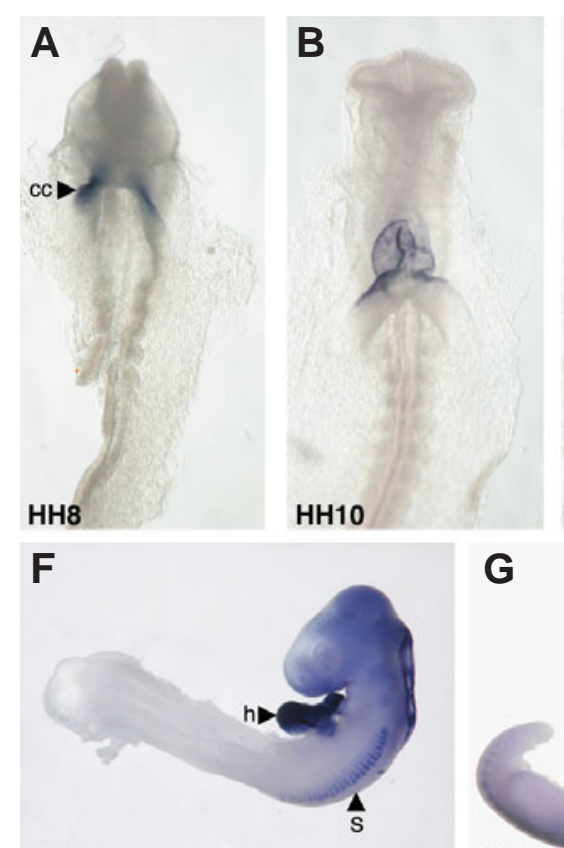

HH17

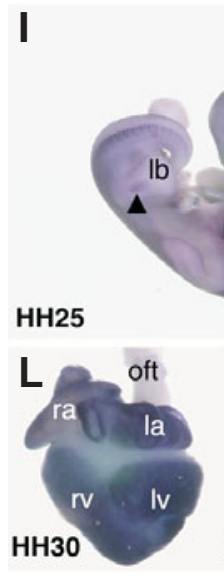

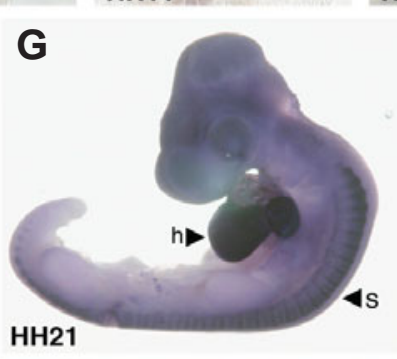

that $C h a p B$ is the predominant isoform in embryonic and fetal stages (Beqqali et al., 2010). In order to determine whether Chap mRNA expression was comparable with protein expression, we performed immunohistochemistry with an antibody that specifically recognizes CHAP (both a and b isoforms). In E12.5 embryos, CHAP protein was detected in heart, trunk muscle masses and muscles of the tongue, eyes and jaws, which was comparable to Chap mRNA expression (Fig. 4 C,D). Furthermore, a striated expression pattern for CHAP, as expected for Z-disc proteins, was observed in muscle cells (Fig. 4E), confirming the specificity of the antibody staining.

\section{Expression of CHAP in adult skeletal muscle}

We have previously shown that both $\mathrm{CHAPa}$ and $\mathrm{CHAPb}$ are expressed in adult mouse heart and skeletal muscle (Beqqali et al., 2010). Here, we investigated the expression of CHAP in adult skeletal muscles in more detail. Skeletal muscle can be divided into slow (type I) and fast (type II) twitch fibers. To determine the expression levels of both Chap isoforms, we isolated slow (soleus) and fast (gastrocnemius) muscles of adult mice. qPCR analysis of these tissues showed that both ChapA (Fig. 5, black bars) and ChapB (Fig. 5, white bars) were expressed higher in soleus than
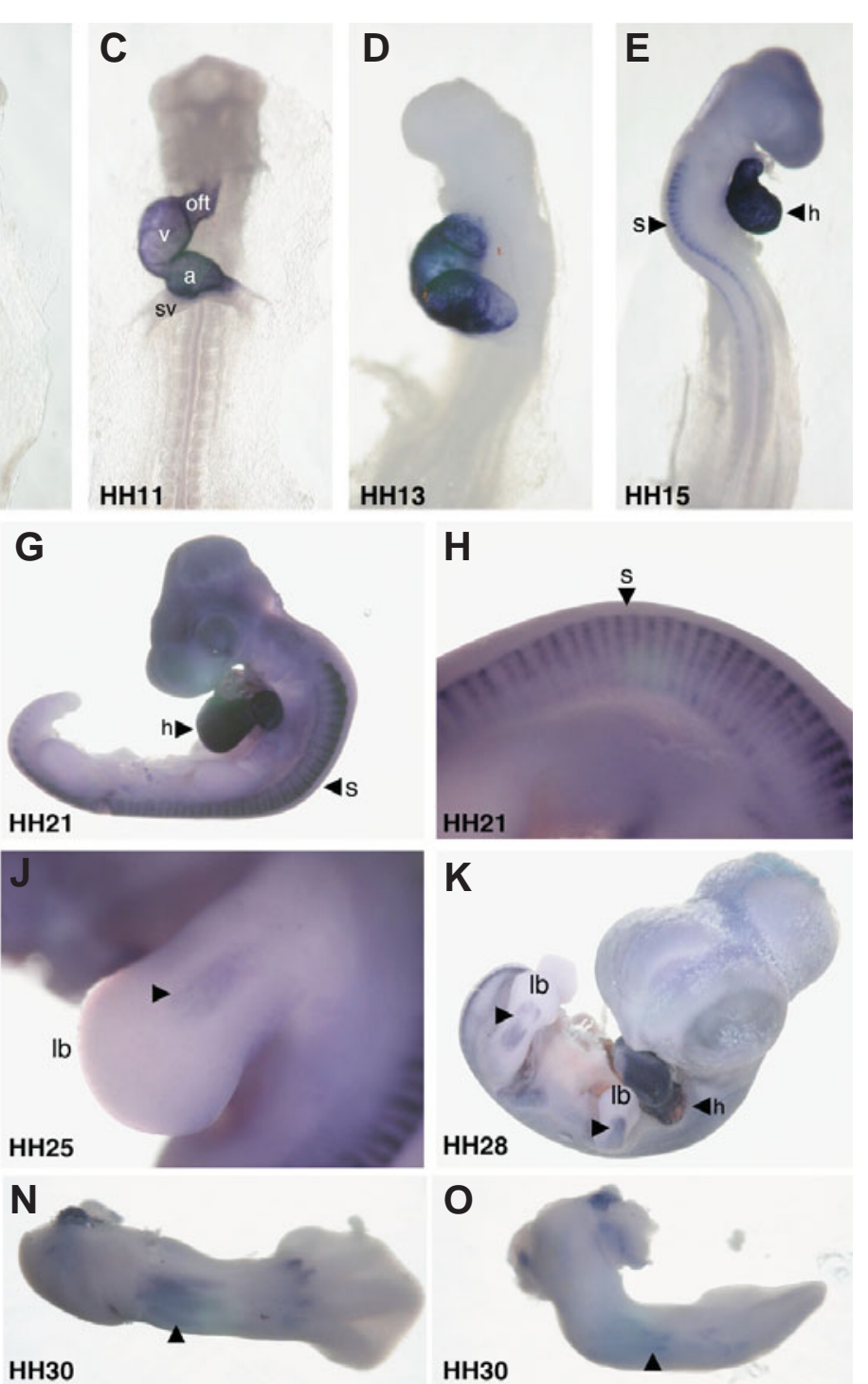


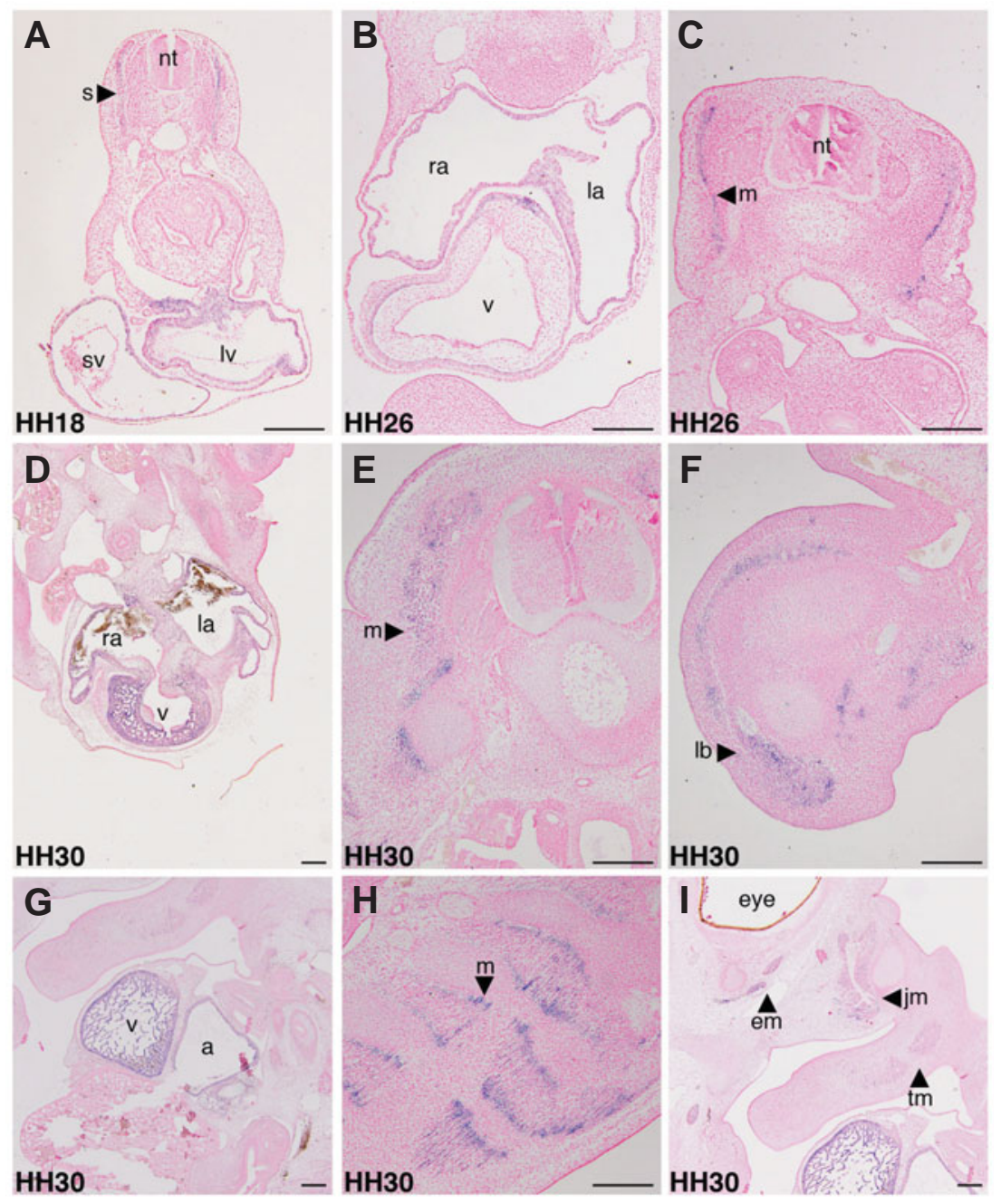

Fig. 3. In situ hybridization of CHAP chick on sections. (A-I) In situ hybridization with digoxigenin-UTP labeled CHAP riboprobe was performed on sections of chick embryos on stages HH18 (A), HH26 (B,C) and HH30 (D-I). Somites (s), myotome (m), limb buds (Ib), eye muscles (em), jaw muscles (jm) and tongue muscles (tm), sinus venosus (sv), left ventricle (IV), ventricle (v), left atrium (la), right atrium (ra), atrium (a), neural tube (nt). Scale bars: $250 \mu \mathrm{m}$

in gastrocnemius. Expression of ChapA was approximately 10 -fold higher than ChapB expression in both soleus and gastrocnemius. To confirm expression at the protein level, we performed CHAP antibody staining in combination with myosin, a slow muscle fiber marker, on sections of mouse soleus and gastrocnemius. Indeed, CHAP protein was expressed in both soleus and gastrocnemius muscle cells (Fig. 6), confirming the results of the qPCR. Although Chap mRNA levels were approximately 3 -fold higher in soleus muscle, immunofluorescence did not show obvious differences for CHAP protein expression between soleus and gastrocnemius muscles. However, more quantitative proteins assays would be necessary to confirm this.

\section{CHAP is expressed in smooth muscle cells}

We have previously observed CHAP expression at the Z-disc in cardiomyocytes of adult mice. More detailed examination indicated CHAP expression in smooth muscle cells. To confirm this observation we performed immunostainings for CHAP and $\alpha$-smooth muscle actin (ASMA), a smooth muscle marker (but also expressed in cardiomyocytes during early cardiac development), on sections of embryonic (E17.5), adult mouse heart, carotid arteries and aorta. We observed that besides expression in cardiomyocytes in E17.5 mouse embryos CHAP was also expressed in the vena cava, but not in the aorta (data not shown), carotid arteries (Fig. 7A) and subclavian artery (Fig. 7 B,C). A higher magnification (Fig. 7 B,C) showed that CHAP is expressed in a striated pattern and co-localized with ASMA-positive cells, suggesting that CHAP is expressed in cardiomyocytes from the vena cava. It has been demonstrated previously that cardiomyocytes are present in the tunica media of the vena cava during embryonic development (Vrancken Peeters et al., 1997; Endo et al., 1996). However, in adult tissues CHAP expression was also observed in smooth muscle cells of aorta, carotid arteries and coronary arteries (Fig. 8). Although CHAP and ASMA are expressed in the same cells, the subcellular localization of both proteins is clearly different.

\section{Discussion}

In the present study we identified and characterized chick CHAP which has significant homology to the human and mouse CHAPa isoform and zebrafish Chap-1 and -2, and contains highly conserved motifs such as the $\mathrm{N}$-terminal PDZ domain and the NLS. Expression of both chick CHAP and mouse Chap mRNA and protein were detected in heart and skeletal muscle (such as limbs, jaw, eye and tongue) throughout embryonic development. Furthermore, we demonstrate in the present study that CHAP is also expressed in cardiomyocytes from the vena cava during mouse embryonic development and in smooth muscle cells of aorta, and coronary and carotid arteries of adult mice. It has been shown previously that multipotent cardiovascular progenitor cells, marked by transcription factors Isl-1 and Nkx2.5, have the capacity to differentiate to vascular smooth muscle cells and cardiomyocytes. (Moretti et al., 2006; Sun et al., 2007; Wu et al., 2006). At the present time, it is not clear whether CHAPpositive smooth muscle cells and cardiomyocytes arise from the same cardiovascular progenitor population, or whether CHAP expression is induced in smooth muscle cells which are derived from a different cardiovascular progenitor population.

Conserved predicted motifs, syntenic organization and developmental expression patterns, suggest that CHAP may play an important role during muscle development. Indeed, knockdown of chap by antisense oligonucleotides in zebrafish had shown previously decreased cardiac contractility, defects in cardiac looping and myofibrillar disarray in muscle cells during embryonic development (Beqqali et al., 2010). CHAP displays homology to synaptopodin and myopodin, which have both been shown to function in actin/ $\alpha$-actinin binding and /or signaling (Asanuma et al., 2005, 2006; Weins et al., 2001). Whereas synaptopodin is expressed in kidney and brain in an isoform specific manner (Asanuma et al., 2005), expression of myopodin can be found in smooth, skeletal 

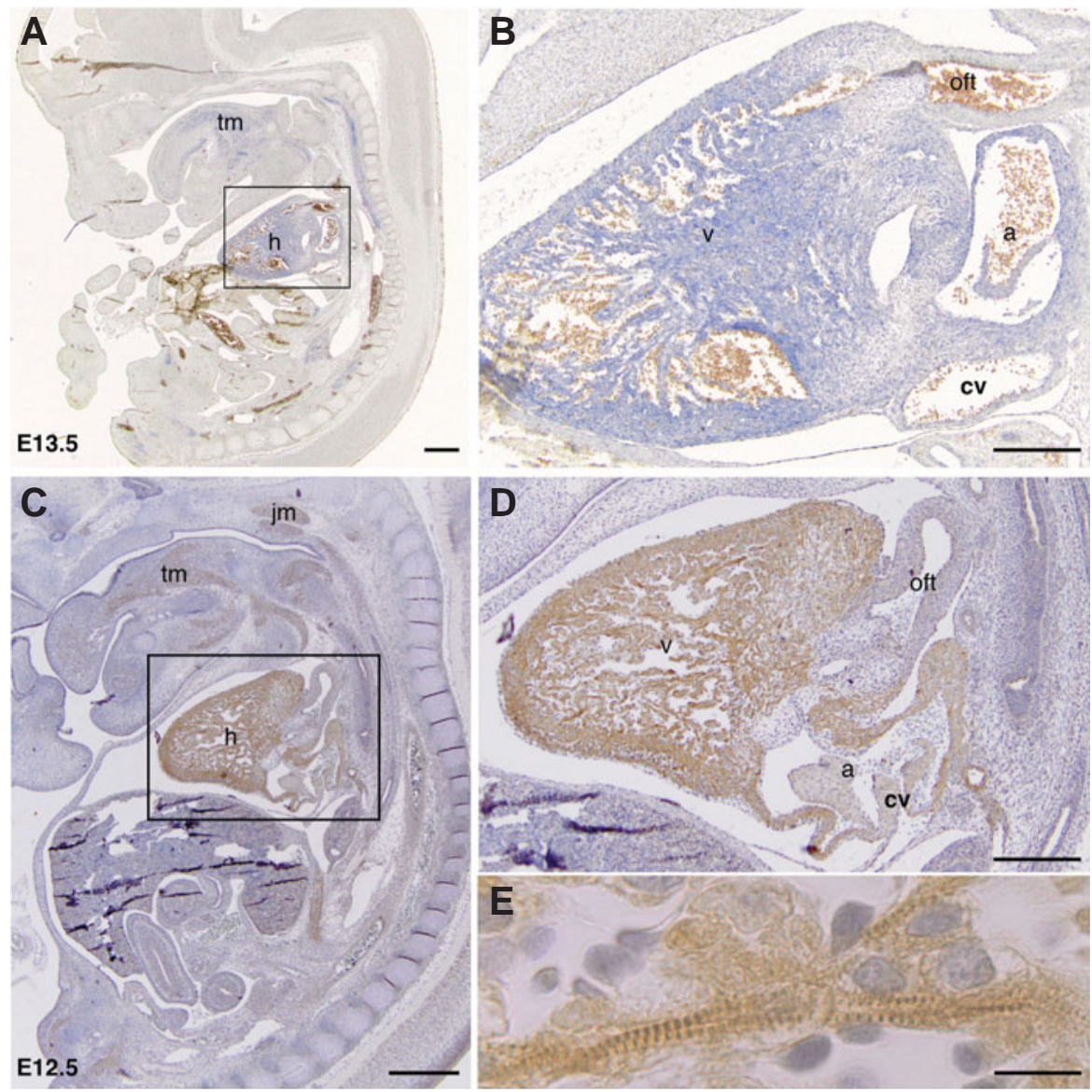

Fig. 4 (Above). Expression and localization of CHAP in mouse embryos. (A,B) Section in situ hybridization with digoxigenin-UTP labeled mouse Chap riboprobe was performed on sagittal sections of E13.5 mouse embryos. A higher magnification of Chap expression in the heart (box in A) is shown in (B). (C-E) Immunostaining for CHAP on sagittal sections of E12.5 mouse embryos (C). Magnification of $C$ (box) is shown in (D). A higher magnification (E) of heart shown in (D) displays a sarcomeric pattern for CHAP. Heart (h), jaw muscle (jm), tongue muscle (tm), ventricle (V), atrium (a), cardinal vein (cv), and outflow tract (oft). Scale bars in (A/C) $500 \mu \mathrm{m}$, in (B/D) $250 \mu \mathrm{m}$ and in (E) $10 \mu \mathrm{m}$.
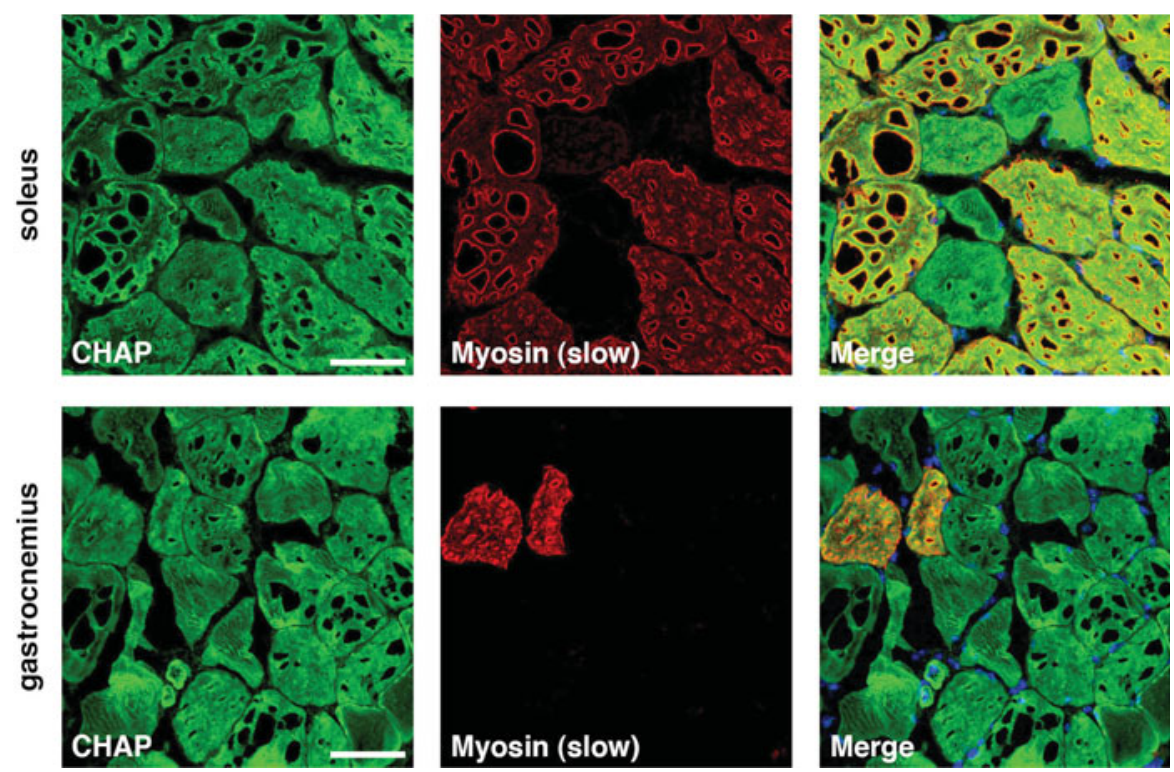

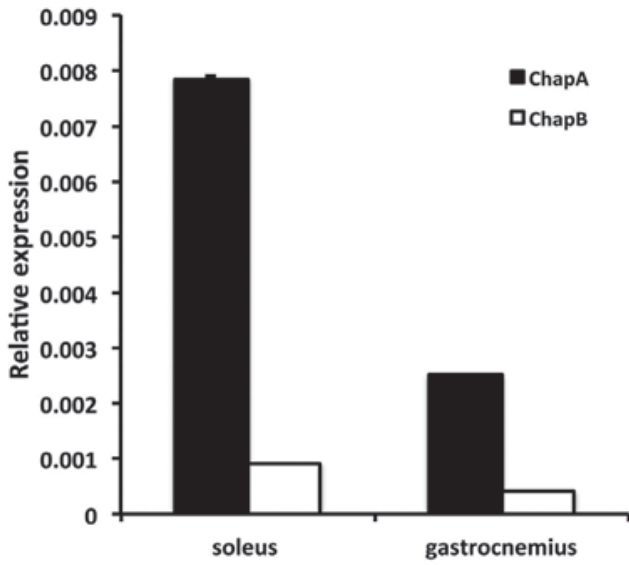

Fig. 5 (Above). ChapA and ChapB expression in adult skeletal muscle. Quantitative real-time $P C R$ expression of ChapA and ChapB mRNA in soleus (slow) and gastrocnemius (fast) muscles. Gapdh used as internal control.

and heart muscle cells, which is comparable to that of CHAP. Like CHAP, myopodin is localized at the Z-disc of striated muscles and is able to translocate to the nucleus (Faul et al., 2007a; De Ganck et al., 2005). It has been shown for myopodin that trafficking between the Z-disc and the nucleus is dependent on PKA and CamKII kinase and calcineurin phosphatase activities (Faul et al., 2007b). Future studies will elucidate whether similar pathways control CHAP trafficking in muscle cells. Furthermore, it would be of interest to study the function and mechanism of action of CHAP in physiological and healthy and pathophysiological situations related to striated and smooth muscle cells. In humans, hypertrophic cardiomyopathy (HCM) and dilated cardiomyopathy (DCM) have been associated with mutations in cytoskeletal and sarcomeric proteins (Sanoudou et al., 2005; Beqqali et al., 2009). In addition, it has been shown in different mouse models that mutations in sarcomeric proteins such as $\alpha$-myosin heavy chain (Georgakopoulos et al., 1999; GeisterferLowrance et al., 1996), myosin binding protein C (Witt et al., 2001; Harris et al., 2002) and cardiac troponin T (Tardiff et al., 1999) can cause HCM-like phenotypes. The sarcomeric protein CHAP may represent a novel candidate gene for screening mutations in DCM and HCM patients.

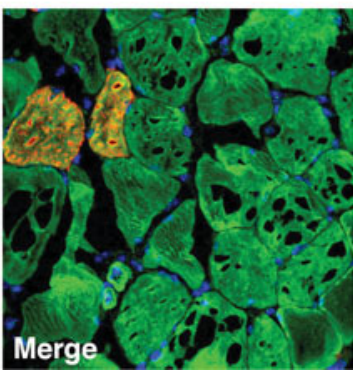

Fig. 6. Expression and localization of CHAP in adult fast and slow muscle. Immunostaining on cross-sections of mouse soleus (slow, upper panel) and gastrocnemius (fast, lower panel) using anti-CHAP (green) and anti-myosin (red) antibodies. Myosin staining (red) was used as a marker for slow muscle fibers, and DAPI was used to stain nuclei (blue). Scale bars: $50 \mu \mathrm{m}$. 

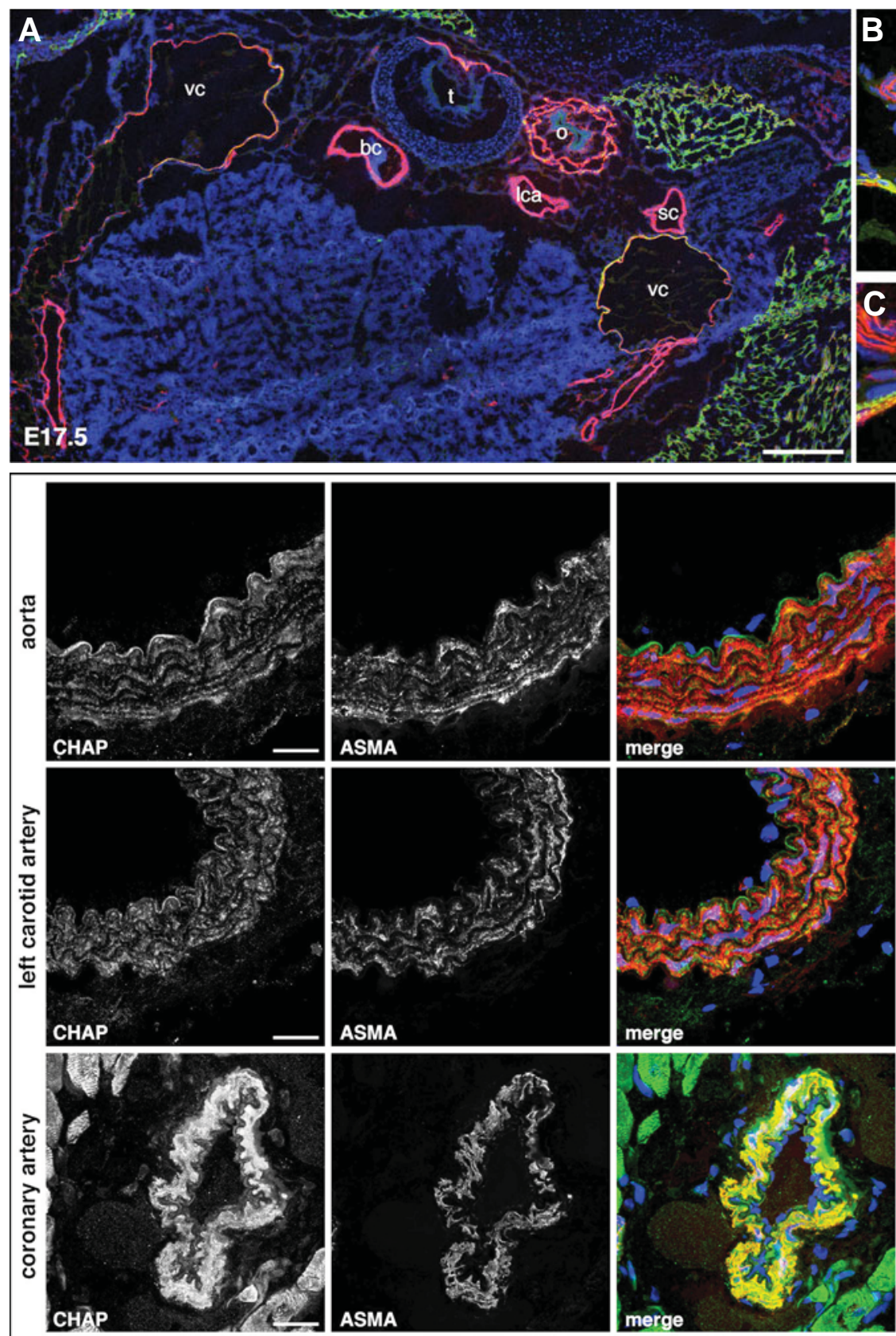

Fig. 8. Expression and localization of CHAP in adult smooth muscle cells. Immunostaining for CHAP (green) and $\alpha$-smooth muscle actin (ASMA; red) on cross-sections of adult mouse aorta, left carotid artery and coronary artery. DAPI was used to stain nuclei (blue). Scale bars: $20 \mu \mathrm{m}$.

\section{Materials and Methods}

\section{Animals}

Swiss mice were intercrossed and females were sacrificed for collection of embryos at different time points (E12.5, 13.5 and 17.5). Fertilized eggs of White Leghorn chicken were incubated at $37^{\circ} \mathrm{C}$ and $80 \%$ humidity. Chick embryos were staged according to the criteria of Hamburger and Hamilton (HH, (Hamburger and Hamilton, 1992)). Mouse (E12.5 and E13.5) and chick embryos were collected and fixed in $4 \%$ paraformaldehyde (PFA; $\mathrm{w} / \mathrm{v}$ ) in phosphate buffered saline (PBS) overnight at $4^{\circ} \mathrm{C}$. Embryos were further processed for whole-mount or section in situ hybridization, or by sequencing.

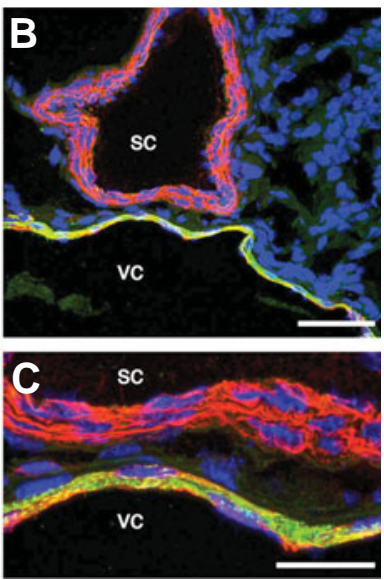

Fig. 7. Co-localization of CHAP with $\alpha$-smooth muscle actin (ASMA) in cardiomyocytes of the vena cava. Immunostaining for CHAP (green) and $\alpha$-smooth muscle actin (red) on cross-sections of E17.5 mouse embryos (AC). DAPI was used to stain nuclei (blue). Vena cava (vc), brachiocephalic artery (bc), left carotid artery (lca), subclavian artery (sc) trachea (t), and oesophagus (0). Scale bars: in (A) $250 \mu \mathrm{m}$, (B) $50 \mu \mathrm{m}$ and (C) $20 \mu \mathrm{m}$.

immunohistochemistry. Processing of mouse embryos for cryosections was adapted from (Bajanca et al., 2004). In brief, E17.5 embryos were fixed in $0.2 \%$ PFA solution containing $4 \%$ sucrose, $0.12 \mathrm{mM} \mathrm{CaCl}_{2} .2 \mathrm{H}_{2} \mathrm{O}$, $0.2 \mathrm{M} \mathrm{Na}_{2} \mathrm{HPO}_{4} \cdot 2 \mathrm{H}_{2} \mathrm{O}, 0.2 \mathrm{M} \mathrm{NaH}_{2} \mathrm{PO}_{4} \cdot \mathrm{H}_{2} \mathrm{O}$ overnight at $4^{\circ} \mathrm{C}$. Next, embryos were washed in the same solution without PFA during the day at $4^{\circ} \mathrm{C}$, followed by $0.24 \mathrm{M}$ phosphate buffer and $30 \%$ sucrose over night at $4{ }^{\circ} \mathrm{C}$. The next day embryos were embedded in Tissue-Tek (Sakura Finetek) on dry ice and stored at $-20^{\circ} \mathrm{C}$ until sectioning. Organs of adult Swiss mice were isolated, rinsed in PBS and processed for RNA isolation and cryosectioning.

\section{RNA isolation and cDNA synthesis}

Total RNA from chick hearts of stage 19, 21 and 27 was isolated using Trizol reagent (Invitrogen), followed by chloroform extraction and ethanol precipitation. Subsequently mRNA was reverse transcribed into cDNA using Superscript II (Invitrogen) and random primers according to the suppliers' protocol.

For qPCR analysis, total RNA was isolated from gastrocnemius and soleus muscles of adult Swiss mice using Trizol reagent as describe above, followed by DNase-treatment (DNA-free, Ambion) and conversion to cDNA using iScript cDNA synthesis kit according to the suppliers' protocol (Bio-Rad). qPCR was performed using the CFX96 Real-Time PCR detection system (Bio-Rad). The following primers were used: ChapA (sense: 5'-GAGGAGGTGCAGGTCACATT-3'; antisense: 5'-CTGAAGAGCCTGGGAAACAG-3'), ChapB (sense: 5'-CCGCCGCTTCTTAAACATAA-3 antisense: 5'-GGCTTTAAAGGGCCTTGG-3') and as reference gene Gapdh (sense: 5'-GTTTGTGATGGGTGTGAACCAC-3', antisense:5'-CTGGTCCTCAGTGTAGCCCAA-3'). Data were analyzed with Bio-Rad CFX Manager.

\section{Cloning of full-length chick CHAP}

Using the mouse and human CHAP coding sequences, a chick CHAP orthologue (XM_421618) was identified after BLAST search. Full-length cDNA of chick $C H A P$ was amplified by PCR (multiple clones) from chicken $\mathrm{HH} 19-27$ hearts and subsequently cloned into the PCRII-TOPO vector (Invitrogen). The full-length coding sequence of chick $\mathrm{CHAP}$ was confirmed

\section{Whole- mount and section in situ hybridization}

RNA probes were generated from PCR products cloned into pCRII-TOPO using T7 or SP 6 primers. For generation of a chick CHAP probe template, 
the following primers were used: sense 5'- GGTCTCCССTTTCTCACCTC-3' and antisense 5'-CACCACAAACTTGTCCATGC-3' (PCR product: $810 \mathrm{bp}$ ). Probes for detection of mouse Chap were generated as described previously (Beqqali et al., 2010). Probes were digoxigenin-labeled according to the suppliers' protocol (Roche Applied Science). For in situ hybridization $10 \mu \mathrm{m}$ sections of chick or mouse embryos were mounted on superfrost slides (Menzel). In situ hybridization was performed as previously described (Moorman et al. 2001). Whole-mount in situ hybridization was performed as previously described (Nieto et al., 1992).

\section{Immunofluorescence}

Mouse embryos and adult tissues were sectioned ( $5 \mu \mathrm{M})$ and mounted on starfrost slides (Knittel). Antibody staining on cryo-sections was performed as previously described (van Laake et al., 2007). Primary antibodies were as follows: anti-CHAP (rabbit, 1:50, custom made by Eurogentec), anti-myosin (mouse, 1:1000, NOQ7.5.4D, Sigma-Aldrich Chemie) and anti- $\alpha$-smooth muscle actin (mouse, 1:500, 1A4, Sigma-Aldrich Chemie). Secondary antibodies were as follows: Cy-3 conjugated anti-mouse (1:250, Jackson Immunoresearch Laboratories) and Alexa488 conjugated anti-rabbit (1:200, Invitrogen). Cell nuclei were stained with DAPI (Molecular Probes)

\section{Immunohistochemistry}

Mouse embryos (E12.5) were sectioned $(5 \mu \mathrm{M})$ and mounted on starfrost slides (Knittel). Antigen retrieval was performed by microwave heating of tissue sections in citrate buffer $(\mathrm{pH} 6)$. Endogenous peroxidase was blocked by incubating the slides in $0.3 \% \mathrm{H}_{2} \mathrm{O}_{2}$ in PBS. Sections were incubated overnight with CHAP antibody $(1: 2000)$ at room temperature. Biotin-conjugated goat anti-rabbit (BA-1000, Vector Labs) was used as secondary antibody. Subsequently, the sections were incubated with Vectastain ABC staining kit (PK-6100, Vector Labs) for $45 \mathrm{~min}$. Slides were rinsed in PBS and Tris/Maleate ( $\mathrm{pH}$ 7.6). 3-3diaminobenzidine tetrahydrochloride (D5637, Sigma-Aldrich) was used as chromogen and Mayer's hematoxylin as counterstaining. Finally, all slides were dehydrated and mounted with Entellan (Merck).

\section{Acknowledgements}

We thank C. van Munsteren and L. Wisse for technical assistance. This study was supported by the Netherlands Heart Foundation grant 2006B209 (W.E.), and by European Community's Sixth Framework Programme contract ('HeartRepair') LSHM-CT-2005-018630 (A.B.).

\section{References}

ASANUMA K, KIM K, OH J, GIARDINO L, CHABANIS S, FAUL C, REISER J and MUNDEL P. (2005). Synaptopodin regulates the actin-bundling activity of alphaactinin in an isoform-specific manner. $J$ Clin Invest 115:1188-1198.

ASANUMA K, YANAGIDA-ASANUMA E, FAUL C, TOMINO Y, KIM K and MUNDEL P. (2006). Synaptopodin orchestrates actin organization and cell motility via regulation of RhoA signalling. Nat Cell Biol 8:485-491.

BAJANCA F, LUZ M, DUXSON MJ and THORSTEINSDOTTIR S. (2004). Integrins in the mouse myotome: developmental changes and differences between the epaxial and hypaxial lineage. Dev Dyn 231:402-415.

BEQQALI A, KLOOTS J, WARD-VAN OOSTWAARD D, MUMMERY C and PASSIER R. (2006). Genome-wide transcriptional profiling of human embryonic stem cells differentiating to cardiomyocytes. Stem Cells 24:1956-1967.

BEQQALI A, MONSHOUWER-KLOOTS J, MONTEIRO R, WELLING M, BAKKERS J, EHLER E, VERKLEIJ A, MUMMERY C and PASSIER R. (2010). CHAP is a newly identified Z-disc protein essential for heart and skeletal muscle function. $J$ Cell Sci 123:1141-1150.

BEQQALIA, VAN ELDIK W, MUMMERY C and PASSIER R. (2009). Human stem cells as a model for cardiac differentiation and disease. Cell Mol Life Sci 66:800-813.

DE GANCKA, HUBERTT, VAN IK, GEELEN D, VANDEKERCKHOVEJ, DE CORTEV and GETTEMANS J. (2005). Amonopartite nuclear localization sequence regulates nuclear targeting of the actin binding protein myopodin. FEBS Lett579:6673-6680.

ENDO H, OGAWA K, KUROHMARU M and HAYASHI Y. (1996). Development of cardiac musculature in the cranial vena cava of rat embryos. Anat Embryol (Berl) 193:501-504.

FAUL C, DHUME A, SCHECTER AD and MUNDEL P. (2007b). Protein kinase A, $\mathrm{Ca} 2+/$ calmodulin-dependent kinase II, and calcineurin regulate the intracellular trafficking of myopodin between the Z-disc and the nucleus of cardiac myocytes. Mol Cell Biol 27:8215-8227.

FAUL C, DHUME A, SCHECTER AD and MUNDEL P. (2007a). Protein kinase A $\mathrm{Ca} 2+/$ calmodulin-dependent kinase II, and calcineurin regulate the intracellular trafficking of myopodin between the Z-disc and the nucleus of cardiac myocytes. Mol Cell Biol 27:8215-8227.

GEISTERFER-LOWRANCEAA, CHRISTEM, CONNERDA, INGWALLJS, SCHOEN FJ, SEIDMAN CE and SEIDMAN JG. (1996). A mouse model of familial hypertrophic cardiomyopathy. Science 272:731-734.

GEORGAKOPOULOS D, CHRISTE ME, GIEWAT M, SEIDMAN CM, SEIDMAN JG and KASS DA. (1999). The pathogenesis of familial hypertrophic cardiomyopathy: early and evolving effects from an alpha-cardiac myosin heavy chain missense mutation. Nat Med 5:327-330.

HAMBURGER V and HAMILTON HL. (1992). A series of normal stages in the development of the chick embryo. 1951. Dev Dyn 195:231-272.

HARRIS SP, BARTLEYCR, HACKERTA, MCDONALD KS, DOUGLASPS, GREASER ML, POWERS PA and MOSS RL. (2002). Hypertrophic cardiomyopathy in cardiac myosin binding protein-C knockout mice. Circ Res 90:594-601.

LANGE S, EHLER E and GAUTEL M. (2006). From A to $Z$ and back? Multicompartment proteins in the sarcomere. Trends Cell Biol 16:11-18.

MOORMAN AF, HOUWELING AC, DE BOER PA and CHRISTOFFELS VM. (2001). Sensitive nonradioactive detection of mRNA in tissue sections: novel application of the whole-mount in situ hybridization protocol. $J$ Histochem Cytochem 49:1-8.

MORETTI A, CARON L, NAKANO A, LAM JT, BERNSHAUSEN A, CHEN Y, QYANG Y, BU L, SASAKI M, MARTIN-PUIG S, SUN Y, EVANS SM, LAUGWITZ KL and CHIEN KR. (2006). Multipotent embryonic isl1+ progenitor cells lead to cardiac, smooth muscle, and endothelial cell diversification. Cell 127:1151-1165.

NIETO MA, BENNETT MF, SARGENT MG and WILKINSON DG. (1992). Cloning and developmental expression of Sna, a murine homologue of the Drosophila snail gene. Development 116:227-237.

SANOUDOU D, VAFIADAKI E, ARVANITIS DA, KRANIAS E and KONTROGIANNIKONSTANTOPOULOS A. (2005). Array lessons from the heart: focus on the genome and transcriptome of cardiomyopathies. Physiol Genomics 21:131-143.

SUN Y, LIANG X, NAJAFI N, CASS M, LIN L, CAI CL, CHEN J and EVANS SM. (2007). Islet 1 is expressed in distinct cardiovascular lineages, including pacemaker and coronary vascular cells. Dev Biol 304:286-296.

TARDIFF JC, HEWETT TE, PALMER BM, OLSSON C, FACTOR SM, MOORE RL, ROBBINS $\mathrm{J}$ and LEINWAND LA. (1999). Cardiac troponin T mutations result in allele-specific phenotypes in a mouse model for hypertrophic cardiomyopathy. $J$ Clin Invest 104:469-481.

VAN LAAKE LW, PASSIER R, MONSHOUWER-KLOOTS J, NEDERHOFF MG WARD-VAN OOSTWAARD D, FIELD LJ, VAN ECHTELD CJ, DOEVENDANS PA and MUMMERY CL. (2007). Monitoring of cell therapy and assessment of cardiac function using magnetic resonance imaging in a mouse model of myocardial infarction. Nat Protoc 2:2551-2567.

VRANCKEN PEETERS MP, GITTENBERGER-DE GROOT AC, MENTINK MM, HUNGERFORD JE, LITTLE CD and POELMANN RE. (1997). Differences in development of coronary arteries and veins. Cardiovasc Res 36:101-110.

WEINS A, SCHWARZ K, FAUL C, BARISONI L, LINKE WA and MUNDEL P. (2001). Differentiation- and stress-dependent nuclear cytoplasmic redistribution of myopodin, a novel actin-bundling protein. J Cell Biol 155:393-404.

WITT CC, GERULL B, DAVIES MJ, CENTNER T, LINKE WA and THIERFELDER L. (2001). Hypercontractile properties of cardiac muscle fibers in a knock-in mouse model of cardiac myosin-binding protein-C. J Biol Chem 276:5353-5359.

WU SM, FUJIWARA Y, CIBULSKY SM, CLAPHAM DE, LIEN CL, SCHULTHEISS TM, ORKIN SH. (2006). Developmental origin of a bipotential myocardial and smooth muscle cell precursor in the mammalian heart. Cell 127:1137-1150. 


\section{Further Related Reading, published previously in the Int. J. Dev. Biol.}

A conserved MRF4 promoter drives transgenic expression in Xenopus embryonic somites and adult muscle Timothy J. Hinterberger

Int. J. Dev. Biol. (2010) 54: 617-625

TGFbeta3 is expressed in differentiating muscle of the embryonic mouse tongue Akira Yamane, Tadayoshi Fukui, Takeo Suga, Ryohei lida and Mitsuhiko Morito Int. J. Dev. Biol. (2010) 54: 221-226

Signalling molecules involved in mouse bladder smooth muscle cellular differentiation Benchun Liu, Dongxiao Feng, Guiting Lin, Mei Cao, Yuet Wai Kan, Gerald R. Cunha and Laurence S. Baskin

Int. J. Dev. Biol. (2010) 54: 175-180

S. macrurus myogenic regulatory factors (MRFs) induce mammalian skeletal muscle differentiation; evidence for functional conservation of MRFs

Hyun-Jung Kim, Robert Güth, Colleen B. Jonsson and Graciela A. Unguez

Int. J. Dev. Biol. (2009) 53: 993-1002

Embryonic development of the proepicardium and coronary vessels

Anna Ratajska, Elzbieta Czarnowska and Bogdan Ciszek

Int. J. Dev. Biol. (2008) 52: 229-236

Distinct patterns of MMP-9 and MMP-2 activity in slow and fast twitch skeletal muscle regeneration in vivo

Malgorzata Zimowska, Edyta Brzoska, Marta Swierczynska, Wladyslawa Streminska and Jerzy Moraczewski

Int. J. Dev. Biol. (2008) 52: 307-314

The Australian lungfish (Neoceratodus forsteri) - fish or amphibian pattern of muscle development?

Agata Kacperczyk and Malgorzata Daczewska

Int. J. Dev. Biol. (2008) 52: 279-286

ISI Impact Factor $(2010)=2.86$
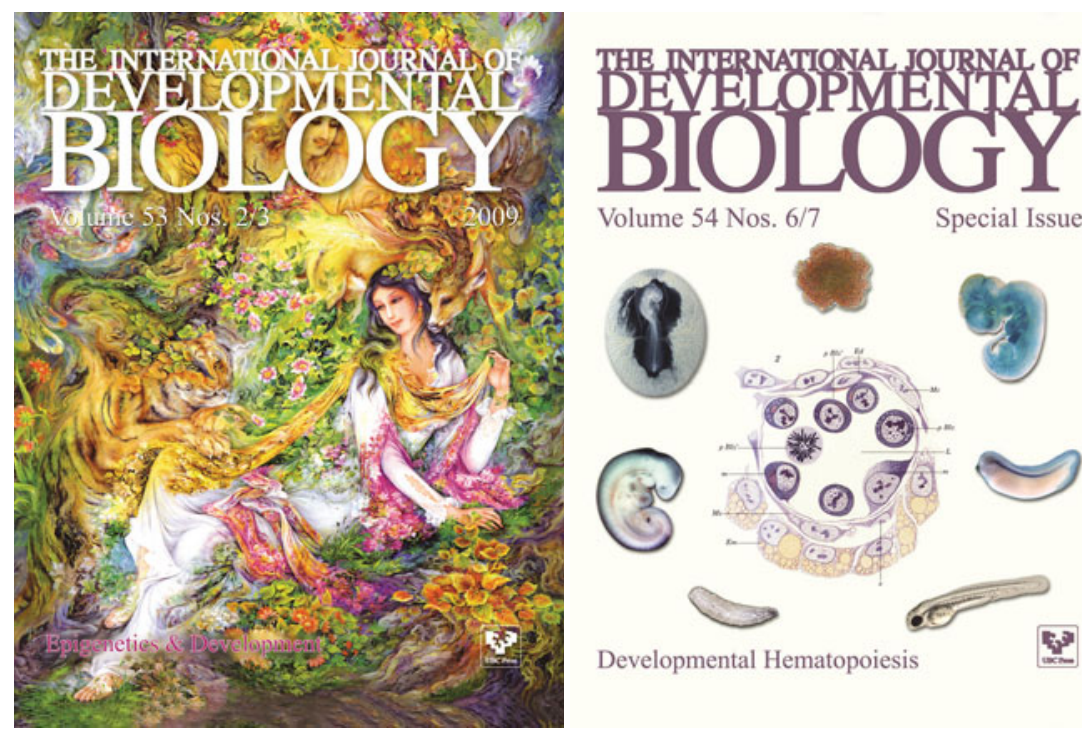

Volume 54 Nos. $6 / 7$

Special Issue
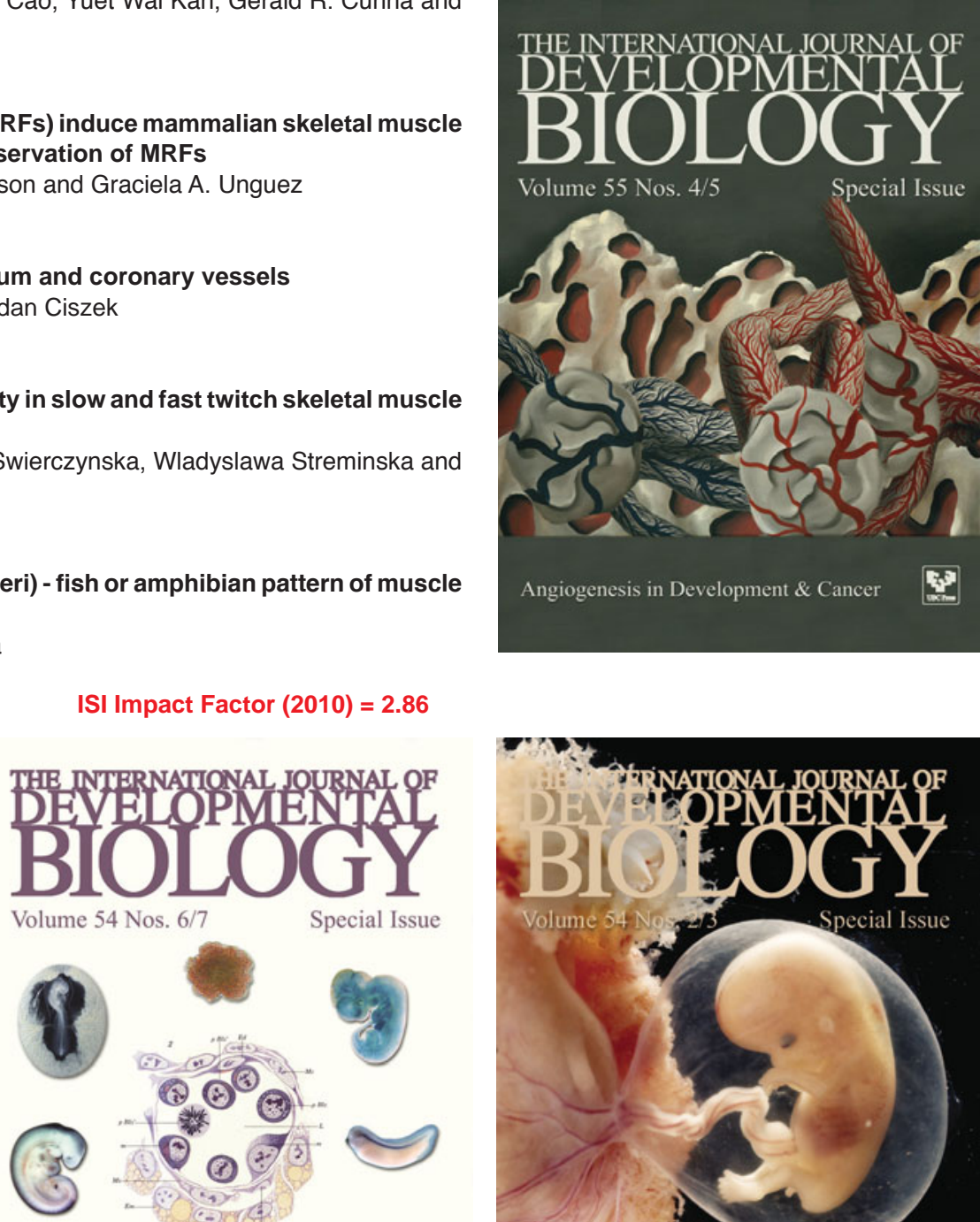

THE INTERNATIONAL JOURNAL OF

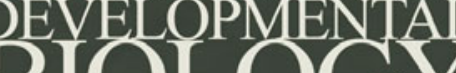

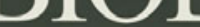

olume 55 Nos. 4

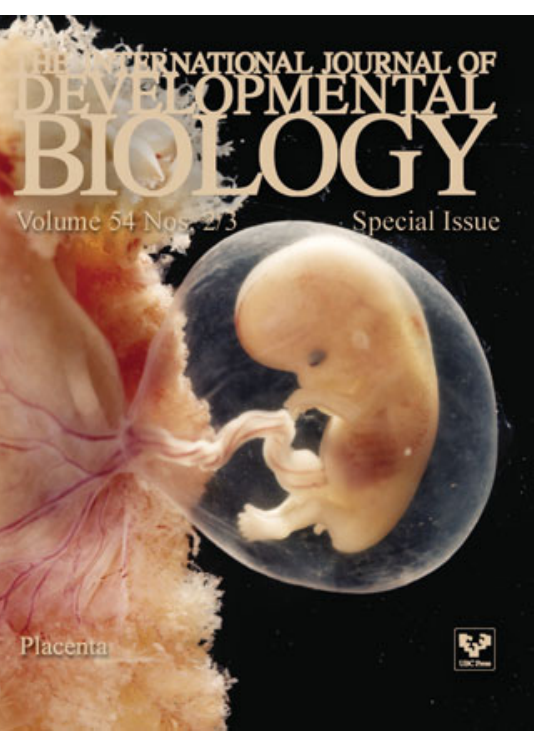

Developmental Hematopoiesis

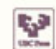

